\title{
Early administration of amatuximab suppresses liver metastasis of mesothelin-expressing pancreatic cancer cells and enhances gemcitabine sensitivity in a xenograft mouse model
}

\section{Yuki Fujii ( $\nabla$ dr-big1023@hotmail.co.jp )}

Hokkaido University Graduate School of Medicine: Hokkaido Daigaku Daigakuin Igakuin https://orcid.org/0000-0002-7742-6937

Hirofumi Kamachi

Hokkaido University Graduate School of Medicine: Hokkaido Daigaku Daigakuin Igakuin Fumihiko Matsuzawa

Hokkaido University Graduate School of Medicine: Hokkaido Daigaku Daigakuin Igakuin

Tatsuzo Mizukami

Hokkaido University Graduate School of Medicine: Hokkaido Daigaku Daigakuin Igakuin

Nozomi Kobayashi

Hokkaido University Graduate School of Medicine: Hokkaido Daigaku Daigakuin Igakuin

Moto Fukai

Hokkaido University Graduate School of Medicine: Hokkaido Daigaku Daigakuin Igakuin

Akinobu Taketomi

Hokkaido University Graduate School of Medicine: Hokkaido Daigaku Daigakuin Igakuin

\section{Research Article}

Keywords: mesothelin, amatuximab, pancreatic cancer, liver metastasis, c-Met, AKT

Posted Date: March 19th, 2021

DOl: https://doi.org/10.21203/rs.3.rs-322672/v1

License: (c) (i) This work is licensed under a Creative Commons Attribution 4.0 International License. Read Full License 


\section{Abstract}

Amatuximab is a promising therapeutic antibody targeting mesothelin, a $40-\mathrm{kDa}$ glycoprotein that is highly expressed in pancreatic cancer. We investigated the effectiveness of early amatuximab treatment, imitating an adjuvant chemotherapy setting, and combination therapy with amatuximab and gemcitabine in liver metastasis of pancreatic cancer. Liver metastasis mouse models were established in 8-week-old male BALB/c nu/nu mice using the hemisplenic injection method. Tridaily amatuximab monotherapy or combination with gemcitabine was administered to the liver metastasis mouse model before metastatic lesions had formed huge masses. Gaussia luciferase-transfected AsPC-1 was used as a mesothelinoverexpressing pancreatic cancer cell line. The amount of liver metastases and the serum luciferase activity were significantly lower in the treatment groups than those in the control IgG group. Notably, the anti-tumor activity of gemcitabine was synergically enhanced by combination therapy with amatuximab. Furthermore, western blotting revealed that the high expression of phosphorylated c-Met and AKT in liver metastatic lesions treated with gemcitabine monotherapy was canceled by its combination with amatuximab. This result indicated that the observed synergic therapeutic effect may have occurred as a result of the inhibitory effect of amatuximab on the phosphorylation of c-Met and AKT, which were promoted by exposure to GEM. In conclusion, our study revealed that early administration of amatuximab alone or in combination with GEM significantly suppressed the liver metastases of mesothelin-expressing pancreatic cancer cells. A phase II clinical trial of amatuximab as part of an adjuvant chemotherapy regimen for resected pancreatic cancer is expected.

\section{Introduction}

Pancreatic ductal adenocarcinoma (PDAC) is the fourth most common in the United States [1]. Surgical resection remains the only potential curative treatment for PDAC, although the prognosis of PDAC is still poor with a 5 -year overall survival (OS) rate of approximately $20 \%$ even after curative surgical resection [2-5]. With the development of adjuvant chemotherapy, including gemcitabine (GEM)- or fluorouracil-based regimens, the prognosis of PDAC has improved to some extent, but the outcome remains unsatisfactory [6-10]. On the other hand, liver recurrence tends to occur earlier than the other recurrences, showing the worst prognosis in several large retrospective studies, which indicates the clinical importance of controlling metastatic liver lesions for further improvement of patient survival [1113].

Mesothelin (MSLN) is a 40-kDa glycophosphatidylinositol-linked cell surface glycoprotein [14-16]. Although MSLN is normally expressed on human mesothelium, it is highly expressed in several malignant tumors, including malignant mesothelioma[14], ovarian cancer[14,17], and pancreatic cancer [18,19]. In these cancers, MSLN may have an important role in cell-to-cell and cell-to-mesothelium adhesion by binding to MUC16 (CA125)[20,21], and functions to stimulate cell proliferation, invasion, migration, and chemoradioresistance [16,22-27]. Moreover, higher expression of MSLN has been associated with poorer prognosis in various types of neoplasms [18,28-33]. 
Amatuximab is a chimerized high-affinity monoclonal lgG1/k antibody targeting MSLN [34]. It blocks the binding of MSLN to MUC16, resulting in the inhibition of heterotypic cell adhesion, and kills MSLNpositive tumor cells via antibody-dependent cellular cytotoxicity (ADCC) [34,35]. Although a previous study showed preferable anti-tumor activity of amatuximab alone or in combination with GEM in pancreatic cancer in in vitro and in vivo studies, a phase II trial (NCT00570713) failed to demonstrate the superiority of combination therapy with amatuximab and GEM compared with single GEM therapy in the survival of patients with unresectable advanced PDAC. Insufficient drug delivery could explain this unfavorable result, such as a negative correlation between tumor size and tumor uptake of amatuximab and/or heterogenous distribution of monoclonal antibody to a bulky pancreatic cancer [36-40]. Furthermore, because MSLN promotes anchorage-independent growth and prevents anoikis[27], any cytotoxic effects of amatuximab may be maximized by administering it before a huge mass is formed by the cancer cells. We previously reported the remarkable anti-adhesive effect of amatuximab in pancreatic cancer cells, creating sherbet-like aggregates of cancer cells in the ascites of a xenograft peritoneal dissemination mouse model, and showed that early administration of amatuximab enhanced the sensitivity of GEM, leading to a significant reduction in tumor burden [41]. Thus, we hypothesized that amatuximab could still be a promising therapeutic agent in an adjuvant chemotherapy setting. However, the effectiveness of amatuximab in metastatic pancreatic cancer remains unknown. Here, we investigated the effectiveness of early amatuximab treatment on liver metastasis of pancreatic cancer and demonstrated that the anti-tumor activity was enhanced by combination therapy with amatuximab and GEM using a xenograft mouse model.

\section{Materials And Methods}

\section{Cell Culture}

AsPC-1, MIA-PaCa-2, and Panc-1 were obtained in January 2014 and Capan-2 was obtained in May 2019 from the American Type Culture Collection (Manassas, VA, USA). ATCC uses morphology-, karyotyping-, and polymerase chain reaction-based approaches to confirm the identity of human cell lines. All cells were stored in liquid nitrogen as original stocks and used for fewer than 3 months after resuscitation from cryopreservation. They were maintained in RPMI-1640 or DMEM supplemented with $10 \%$ fetal bovine serum, $100 \mathrm{U} / \mathrm{ml}$ penicillin, and $100 \mu \mathrm{g} / \mathrm{ml}$ streptomycin, and were cultured in a humidified incubator containing $5 \% \mathrm{CO} 2$ in air at $37^{\circ} \mathrm{C}$.

\section{Reagents}

Amatuximab was obtained from Morphotek (Exton, PA, USA). Control whole molecule human IgG and GEM were purchased from Wako Pure Chemical Industries, Ltd. (Osaka, Japan) and Eli Lilly (Indianapolis, IN, USA), respectively.

\section{Western blot analysis and antibodies}


Cells were seeded at a density of $1.5 \times 10^{6}$ cells in a 100 -mm dish. After 48 -h incubation at $37^{\circ} \mathrm{C}$, the cells were harvested and washed with phosphate-buffered saline (PBS). For animal samples, resected tissues were cut into minute pieces with scissors and a homogenizer. The samples were homogenized at $4^{\circ} \mathrm{C}$ in Nonidet P40 lysis buffer and a protease inhibitor cocktail (Sigma-Aldrich, St. Louis, MO, USA). After centrifugation, extracted total protein lysates were titrated with Laemmli sample buffer solution (Bio-Rad, Hercules, CA, USA) to a concentration of $1 \mu \mathrm{g} / \mathrm{ml}$. Adjusted protein lysates $(12 \mu \mathrm{g})$ were resolved by electrophoresis and western blot analysis was performed as previously described [41]. Protein bands were quantified by densitometry using ChemiDoc XRSâ (Bio-Rad). Primary antibodies are described in Supplemental Table 1.

\section{Fluorescence-activated cell sorting (FACS) analysis}

Cultured cells were washed and resuspended in PBS. Amatuximab, a primary antibody, was added at $2 \mu \mathrm{g} / \mathrm{ml}$ and cells were incubated for $30 \mathrm{~min}$ on ice. The cells were washed with PBS and incubated with $1 \mu \mathrm{g} / \mathrm{ml}$ FITC goat-anti-human IgG secondary antibody (SouthernBiotech, Birmingham, AL, USA) for 30 min. Finally, they were washed again with PBS and analyzed using a BD FACS Canto ${ }^{\mathrm{TM}}$ II flow cytometer (BD Biosciences, Franklin Lakes, NJ, USA).

\section{Immunohistochemistry}

Immunohistochemistry (IHC) staining was performed using the EnVision FLEX/HRP system (Dako Japan, Tokyo, Japan). In summary, formalin-fixed paraffin-embedded sections were mounted on charged glass slides, deparaffinized, and rehydrated through a graded ethanol series. Blocking was performed with a peroxidase blocking reagent (Dako Japan). The sections were incubated with primary antibody against MSLN (\#TA308767; OriGene Technologies, Rockville, MD, USA) diluted to 1:500 at room temperature for 30 min, and reacted with EnVision ${ }^{\text {TM }}$ FLEX/HRP secondary antibody-coated polymer peroxidase complexes (\#SM802; Dako Japan) for 30 min followed by DAB substrate/chromogen. Finally, slides were counterstained with hematoxylin, dehydrated, and mounted.

\section{Animals}

Seven-week-old male BALB/c nu/nu mice were purchased from Japan SLC, Inc. (Shizuoka, Japan). They were maintained under pathogen-free conditions and allowed to adapt to the environment for 1-2 weeks before experiments. All procedures involving animals and their care were approved by the ethics committee of Hokkaido University and were conducted under institutional guidelines for animal experiments.

\section{Establishment of liver metastasis xenograft mouse model}

General anesthesia was induced by $3 \%$ isoflurane and maintained by $1.75 \%-2 \%$ isoflurane during the procedure. The spleen was exposed by left subcostal incision (Fig. 1A). An avascular area of splenic hilum was dissected, and the spleen was cut into halves through the middle after double-clipping (Figure. 
1B,C). A total of $1^{\prime} 10^{6}$ pancreatic cancer cells suspended in $100 \mu \mathrm{L}$ PBS were injected into the lower half of the spleen and the procedure was paused for $2 \mathrm{~min}$ to allow the cells to travel through the portal vein (Fig. 1D). Then, the lower half of the spleen was removed with the clipping and ligation of proximal blood vessels (Fig. 1E). After intra-abdominal irrigation with $20 \mathrm{~mL}$ normal sterile saline, the incision was sutured and covered with surgical tape. Mice were killed on day 42 and a thorough review was performed to identify any metastasis or abdominal dissemination.

\section{Establishment of AsPC-1-Gluc and substrate preparation}

To estimate the tumor volume in vivo, we generated AsPC-1 cells stably expressing Gaussia luciferase (Gluc), a secretory type of luciferase isolated from the marine copepod Gaussia princeps, as previously reported [41]. Briefly, AsPC-1 cells were transfected with vector carrying a Gluc gene using FuGENE HD Transfection Reagent (Roche, Basel, Switzerland). Stably-transfected cells were selected in 400 g/ml G418 (Sigma-Aldrich) for 3 weeks. Pierce ${ }^{\text {TM }}$ Gaussia Luciferase Glow Assay Kit (Thermo Fisher Scientific, Waltham, MA, USA) was used to calculate Gluc activity according to the manufacturer's instructions.

\section{Correlation between the number of AsPC-1-Gluc cells or total tumor weight of liver metastases and secreted Gluc activity in medium or serum}

AsPC-1-Gluc cells $(5,000,10,000,20,000,30,000,40,000,50,000)$ were seeded in a 96 -well microplate $(n=4)$ and incubated for $24 \mathrm{~h}$. Subsequently, $10 \mu \mathrm{L}$ of the supernatant was collected from each well. In the mouse model, serum was collected from the tail vein with microhematocrit capillary tubes. Ten microliters of these samples were mixed with reagent to calculate the Gluc activity in accordance with the instructions of the luciferase assay kit, as described above.

\section{Evaluation of the effects of single amatuximab treatment in an adjuvant setting in the liver metastasis mouse models}

First, we evaluate the therapeutic effect of single amatuximab on the formation of liver metastases. Amatuximab or control lgG $(60 \mathrm{mg} / \mathrm{kg})$ was administered intravenously every third day to the liver metastasis mouse models $(n=8)$. Treatment was initiated on the day of tumor inoculation. Serum samples were collected from the tail vein every 7 days and mice were killed on day 42 . Liver metastases were dissected from normal liver tissues by microscopy and the total amount of tumor was measured. Additionally, serum Gluc activity was calculated in accordance with the method described above.

\section{Survival analysis of single amatuximab treatment in the liver metastasis mouse models}

Xenograft liver metastasis mouse models were generated with AsPC-1-Gluc cells according to the same method described above. Amatuximab or control lgG $(60 \mathrm{mg} / \mathrm{kg})$ was administered intravenously to mice every third day for the first 3 weeks after tumor inoculation. Mice were killed when massive hemorrhagic ascites or severe cachexia, defined as a $15 \%$ decrease in body weight, was recognized. 


\section{Evaluation of the effect of combination therapy with amatuximab and gemcitabine in liver metastasis mouse models and investigation of biological alterations in liver metastases}

To evaluate the effectiveness of combination therapy with amatuximab and GEM, we modified the treatment model by administering drugs after tumor settlement had been confirmed. In this model, treatment was initiated on day 7 after tumor inoculation. Amatuximab or control lgG $(60 \mathrm{mg} / \mathrm{kg})$ intravenously in combination with or without GEM $(80 \mathrm{~kg} / \mathrm{mg})$ intraperitoneally was administered on days 7,10 , and $13(n=6)$. Serum samples were collected from the tail vein every 7 days and mice were killed on day 20. Whole liver specimens including metastases were fixed with formalin, embedded in paraffin, sliced sequentially at a thickness of $4 \mu \mathrm{m}$, and used for hematoxylin and eosin (H\&E) staining. The metastatic area was calculated using ImageJ software as the total area of five different views that had predominant tumor metastases. The number of tumors was also counted in the same view. Furthermore, serum Gluc activity was calculated in accordance with the method described above. Several pieces of liver metastases obtained at the time of death were collected and analyzed for protein expression by western blotting.

\section{Statistical analysis}

Correlations between two variables were evaluated with Pearson correlation coefficient. The goodness-offit of the model was expressed by $\mathrm{R}^{2}$. Continuous variables were expressed as mean \pm standard error. Differences in continuous variables between two groups and among multiple groups were compared using Student's t-test and the Tukey-Kramer method, respectively. Survival curves were determined using the Kaplan-Meier method, and the log-rank test was used to compare the survival curves of the two groups. The significance level for all statistical testing was set at $p<0.05$. All statistical analyses were performed using JMP Pro 14.0.0 software (SAS Institute Inc., Cary, NC, USA).

\section{Results}

\section{Mesothelin expression among pancreatic cancer cell lines}

We previously reported MSLN expression in four human pancreatic cancer cell lines-AsPC-1, Capan-2, MIA PaCa-2, and Panc-1-by western blotting, IHC, and FACS analysis [41,42]. We reconfirmed MSLN expression in these cells by western blotting, revealing that AsPC-1 and Capan-2 expressed robust levels of MSLN, whereas Panc-1 and MIA PaCa-2 did not (Supplemental Fig. 1). This result was compatible with that obtained in the previous studies. However, all cell lines showed robust, comparable levels of MUC16 expression (Supplemental Fig. 1).

\section{Comparison of liver metastases in pancreatic cell lines}

We established a liver metastasis mouse model using AsPC-1, Capan-2, MIA PaCa-2, and Panc-1 according to the hemisplenic injection method described in the Methods section. Among four pancreatic cancer cell lines, only AsPC-1 formed sufficient volumes of liver metastases by the hemisplenic injection 
method (Fig. 1F,G). H\&E and IHC staining with anti-MSLN antibody demonstrated that multi-centric lesions were broadly identified in the liver (Fig. $1 \mathrm{H}, \mathrm{I})$. Metastases at other sites, such as intra-abdominal lymph nodes, lung, and brain, and abdominal dissemination, were not recognized. The other three cell lines-Capan-2, MIA PaCa-2, and Panc-1-created trivial liver metastasis that were not suitable to be used in further experiments (Supplemental Fig. 2; $n=5$ ).

\section{Establishment of Gaussia luciferase-transfected AsPC-1 cells and correlations between Gluc activity and the number of cells or the amount of liver metastases}

We established an AsPC-1-Gluc cell line to calculate the tumor burden by measuring serum Gluc activity. AsPC-1-Gluc cells expressed equivalent levels of MSLN and MUC16 to wild-type AsPC-1 cells (Supplemental Fig. 3A), and FACS analysis confirmed strong MSLN expression on the surface of both AsPC-1 and AsPC-1-Gluc cells using amatuximab (MORAb-009) (Supplemental Fig. 3B). A significant strong correlation was shown between the number of cells and the Gluc activity in the medium (Supplemental Fig. 3C; $\mathrm{R}^{2}=0.981, P<0.001$ ). Furthermore, the Gluc activity in mouse serum was significantly correlated with the total tumor weight in liver metastasis mouse models (Supplemental Fig. $\left.3 \mathrm{D} ; \mathrm{R}^{2}=0.972, P<0.001\right)$.

Single amatuximab treatment suppressed the development of liver metastases in the xenograft mouse model

Single amatuximab treatment significantly suppressed the formation of liver metastases in xenograft mouse models compared with control IgG (Figure. 2A). The total weight of liver metastases in the amatuximab group was lower than that in the control IgG group $(100.1 \pm 52.4 \mathrm{mg}$ vs. $489.1 \pm 164.9$ $\mathrm{mg}, P=0.041$; Fig. 2B). Additionally, serum Gluc activity in the control IgG group on days 21,35 , and 42 was significantly higher than that in the amatuximab group (Fig. 2C). However, the body weights of the mice in the two groups were equivalent ( $P \geq 0.05$ at all points except on day 14), supporting the tumorspecific targeting activity of amatuximab (Fig. 2D).

\section{Single amatuximab treatment prolonged overall survival in the xenograft mouse model}

Figure 3 shows Kaplan-Meier curves of OS by treatment type. The median OS of mice in the two groups was 54 days in the control IgG group and 61 days in the amatuximab group $(P=0.0256)$. There was no metastasis at other sites of the body and all mice died of tumor embolism in the inferior vena cava causing massive hemolytic ascites.

\section{Combination therapy with amatuximab and gemcitabine synergically suppressed the development of liver metastases formation in the xenograft mouse model}

Representative H\&E staining in the four groups treated with control IgG, amatuximab, control IgG + GEM, and amatuximab + GEM are shown in Figure 4A. On ImageJ analysis, the mean area of metastatic tumors in the amatuximab group, control IgG + GEM group, and amatuximab + GEM group was 
significantly smaller than that in the control $\lg$ group $\left(8.4 \pm 1.2^{\prime} 10^{5} \mu \mathrm{m}^{2}, 8.9 \pm 1.1^{\prime} 10^{5} \mu \mathrm{m}^{2}, 3.4 \pm\right.$ $0.3^{\prime} 10^{5} \mu \mathrm{m}^{2}$, and $22.1 \pm 1.2^{\prime} 10^{5} \mu \mathrm{m}^{2}$, respectively; $P<0.0001$ ) (Fig. 4B). Furthermore, of those three treatment groups, the amatuximab + GEM group had the smallest tumor area, which was statistically significant. Additionally, the mean number of tumors in the three treatment groups was significantly lower than that in the control IgG group $(19.5 \pm 3.0,21.2 \pm 3.5,12.5 \pm 1.7$, and $33.5 \pm 1.5$, respectively; $P<$ 0.0001 ) (Fig. 4C). Serum Gluc activity was significantly lower in the amatuximab + GEM group than in the control group on day $20(10910 \pm 383.6$ relative light unit (RLU) vs. $22566.7 \pm 4610.8 \mathrm{RLU} ; P=0.012)$ (Fig. 4D). The body weight of mice in all groups was equivalent ( $P \geq 0.05$ at all points) (Fig. 4E).

\section{Phosphorylation of c-Met and AKT was inhibited by combination therapy with amatuximab and gemcitabine compared with gemcitabine monotherapy}

In further biological investigations of amatuximab, we focused on the c-Met/PI3K/AKT pathway. Western blotting performed on lysates from metastatic tumors revealed significantly higher expression of phosphorylated c-Met and AKT in the control IgG + GEM group (single GEM treatment group) than in the control group. However, this high expression of phosphorylated c-Met and AKT was inversely decreased by combining GEM with amatuximab, suggesting the inhibitory effect of amatuximab on the phosphorylation of c-Met and AKT. The levels of total c-Met and AKT were equivalent among all four groups (Fig. 5A,B).

\section{Discussion}

To develop an innovative adjuvant chemotherapy approach to inhibit liver metastasis of pancreatic cancer, we performed preclinical evaluation of amatuximab by establishing a liver metastasis xenograft mouse model in which we readily calculated the tumor burden by measuring the serum Gluc activity. Our results revealed that early administration of amatuximab suppressed the liver metastases of MSLNoverexpressing pancreatic cancer cells and prolonged survival in a xenograft mouse model. Additionally, the anti-tumor activity of GEM was synergically enhanced by combination therapy with amatuximab. Furthermore, higher expression of phosphorylated c-Met and AKT in liver metastatic lesions was observed in the GEM group compared with mice treated with control IgG, amatuximab, and amatuximab and GEM combination. This result indicated that the synergic therapeutic effect resulted from the inhibitory effect of amatuximab on the phosphorylation of c-Met and AKT, which was promoted by the exposure to GEM.

Previous preclinical studies reported the effectiveness of single amatuximab on MSLN-expressing cancer cells using a subcutaneous or an abdominal dissemination xenograft mouse model [34,41]. In these studies, amatuximab exhibited synergistic cytotoxicity on MSLN-expressing cancer cells with GEM, while single amatuximab treatment showed trivial or mild anti-tumor activity. Interestingly, the anti-tumor activity of single amatuximab treatment was more robust in this study than that in previous studies. We assume that this discrepancy may have arisen from the differences in drug distribution between the liver and other sites. The biodistribution of amatuximab in mice has been thoroughly investigated by radiological studies, and the liver uptake of amatuximab is larger than that of other gastrointestinal or 
musculoskeletal organs $[39,40]$. Furthermore, liver uptake is correlated positively to tumor size, whereas an inverted correlation between tumor size and blood retention is observed. This suggests that amatuximab has stronger cytotoxicity in smaller liver tumors, supporting the propriety of the early use of amatuximab to prevent liver recurrence. Furthermore, the innate immune environment of nude mice is much different between the liver and other organs. Because amatuximab targets cancers via ADCC activity, the local population of macrophages and natural killer cells may have a critical role in the cytotoxic mechanism of amatuximab. Although whether amatuximab, which is present in $83 \%$ of human and $17 \%$ of murine sequences, can induce ADCC in human pancreatic cancer cells in a nude mouse is unclear, several studies demonstrated that ADCC was stimulated by humanized/chimeric IgG from murine mononuclear cells in human cancerous cells $[43,44]$. Another study illustrated the high affinity of human IgG to murine Fc gamma receptors, which indicated the possibility of human IgG subclasses having similar relative Fc gamma receptor-mediated biological activity in mice, suggesting ADCC involvement in our xenograft mouse model [45]. These findings highlight the robust therapeutic effect of single amatuximab treatment to prevent liver metastasis of pancreatic cancer as well as its clinical application as a promising agent for adjuvant chemotherapy.

Notably, our results suggested that the synergic anti-tumor activity of combined amatuximab and GEM treatment in MSLN-expressing cancer occurred through inhibition of the phosphorylation of c-Met and AKT. The synergic therapeutic effect of amatuximab with GEM was reported in several in vivo studies, but the exact biological mechanism through which amatuximab strengthens chemosensitivity has not yet been thoroughly investigated $[34,41]$. Our recent in vitro study demonstrated that amatuximab suppressed cell invasion and enhanced GEM sensitivity by regulating cancer stemness and by inhibiting phosphorylated c-Met in MSLN-overexpressing pancreatic cancer cell lines [42]. However, activating the $\mathrm{PI} 3 \mathrm{~K} / \mathrm{AKT}$ pathway is considered as a potential mechanism by which pancreatic cancer acquires chemoresistance [46,47]. In this context, Chang et al. reported that MSLN inhibits paclitaxel-induced apoptosis via the PI3K/AKT pathway in a PI3K-dependent manner [23]. These findings underlie our results of the synergic therapeutic effect of combination therapy with amatuximab and GEM to suppress the liver metastases of pancreatic cancer.

In the present study, we could not analyze the immunological reaction caused by amatuximab. Because we established a xenograft model using a human-derived cancer cell line, a chimeric antibody, and a nude mouse, an unnatural immune response may have inevitably occurred in our study. To understand the precise immunological mechanism of amatuximab in cancer cells, the use of a humanized NOD/SCID/gamma(c)(null) mouse is necessary. Furthermore, we only used an AsPC-1 cell-line derived xenograft model. An additional investigation using multiple cell lines or a patient-derived xenograft mouse model will provide us with more practical findings of amatuximab treatment and details of amatuximab-driven molecular pathways.

In conclusion, our study revealed that early administration of amatuximab alone or in combination with GEM suppressed the liver metastases of MSLN-expressing pancreatic cancer cells. In addition to the results of previous studies, which revealed the significant therapeutic effect of amatuximab in 
subcutaneous tumors or abdominal dissemination of pancreatic cancer, our results strongly promote the clinical use of early amatuximab treatment. A phase I clinical study revealed the detailed pharmacokinetics of amatuximab and found it was well tolerated for clinical application[48], and thus a phase II clinical trial of amatuximab as part of an adjuvant chemotherapy regimen for resected PDAC is expected.

\section{Abbreviations}

ADCC, antibody-dependent cellular cytotoxicity; FACS, fluorescence-activated cell sorting; GEM, gemcitabine; Gluc, Gaussia luciferase; H\&E, hematoxylin and eosin; IHC, immunohistochemistry; MSLN, mesothelin; OS, overall survival; PBS, phosphate-buffered saline; PDAC, pancreatic ductal adenocarcinoma; $\mathrm{RLU}$, relative light unit

\section{Declarations}

Ethics approval and consent to participate: This article does not contain any studies with human participants. For this type of study, formal consent is not required. All procedures involving animals and their care were approved by the ethics committee of Hokkaido University and were conducted under institutional and Japanese governmental guidelines for animal experiments.

Consent for publication: All authors consent to the publication of this study.

Availability of data and material: The authors confirm that the data supporting the findings of this study are available within the article and its supplementary materials.

Competing interests: All authors declare that they have no competing interests.

Funding: This study was supported by Japan Society for the Promotion of Science under Grant-in-Aid for Scientific Research C-20K09024.

\section{Authors' contributions:}

Yuki Fujii: Writing - Original Draft, Data Curation, Investigation, Formal Analysis, Visualization. Hirofumi Kamachi: Conceptualization, Methodology, Funding acquisition, Writing - Review \& Editing. Fumihiko Matsuzawa: Investigation, Writing - Review \& Editing. Tatsuzo Mizukami: Investigation, Writing - Review \& Editing. Nozomi Kobayashi: Methodology, Investigation, Resources. Moto Fukai: Resources, Writing Review \& Editing. Akinobu Taketomi: Supervision, Project administration, Writing - Review \& Editing.

\section{Acknowledgements}

We are grateful to Dr. Keiji Furuuchi (Morphotek Inc.) for providing amatuximab from Morphotek Inc. We would like to thank Dr. Hidemitsu Kitamura (Institute for Genetic Medicine, Research Section of Disease Control, Hokkaido University) and Dr. Yutaka Hatanaka (Research Division of Companion Diagnostics, 
Hokkaido University Hospital) for their constructive advice. Finally, we would also like to thank H. Nikki March, PhD, Edanz Group (https://en-author-services.edanz.com/ac) for editing a draft of this manuscript.

\section{COMPLIANCE WITH ETHICAL STANDARDS}

Disclosure of potential conflicts of interest All authors declare that they have no conflicts of interest.

Research involving Human Participants and/or Animals: This article does not contain any studies with human participants. All procedures involving animals and their care were approved by the ethics committee of Hokkaido University and were conducted under institutional and Japanese governmental guidelines for animal experiments.

Informed consent: For this type of study, formal consent is not required.

\section{References}

1. Siegel RL, Miller KD, Jemal A (2019) Cancer statistics, 2019. CA Cancer J Clin 69:7-34. http://doi.org/10.3322/caac.21551.

2. He J, Ahuja N, Makary MA, et al (2014) 2564 resected periampullary adenocarcinomas at a single institution: trends over three decades. HPB (Oxford) 16:83-90. http://doi.org/10.1111/hpb.12078.

3. Katz MH, Wang H, Fleming JB, et al (2009) Long-term survival after multidisciplinary management of resected pancreatic adenocarcinoma. Ann Surg Oncol 16:836-847. http://doi.org/10.1245/s10434008-0295-2.

4. Ferrone CR, Brennan MF, Gonen M, et al (2008) Pancreatic adenocarcinoma: the actual 5-year survivors. J Gastrointest Surg 12:701-706. http://doi.org/10.1007/s11605-007-0384-8.

5. Ferrone CR, Pieretti-Vanmarcke R, Bloom JP, et al (2012) Pancreatic ductal adenocarcinoma: longterm survival does not equal cure. Surgery 152:S43-49. http://doi.org/10.1016/j.surg.2012.05.020.

6. Labori KJ, Katz MH, Tzeng CW, et al (2016) Impact of early disease progression and surgical complications on adjuvant chemotherapy completion rates and survival in patients undergoing the surgery first approach for resectable pancreatic ductal adenocarcinoma - A population-based cohort study. Acta Oncol 55:265-277. http://doi.org/10.3109/0284186X.2015.1068445.

7. Oettle H, Neuhaus P, Hochhaus A, et al (2013) Adjuvant chemotherapy with gemcitabine and longterm outcomes among patients with resected pancreatic cancer: the CONKO-001 randomized trial. JAMA 310:1473-1481. http://doi.org/10.1001/jama.2013.279201.

8. Parikh AA, Maiga A, Bentrem D, et al (2016) Adjuvant Therapy in Pancreas Cancer: Does It Influence Patterns of Recurrence? J Am Coll Surg 222:448-456. http://doi.org/10.1016/j.jamcollsurg.2015.12.031.

9. Ueno $\mathrm{H}$, Kosuge $\mathrm{T}$, Matsuyama $\mathrm{Y}$, et al (2009) A randomised phase III trial comparing gemcitabine with surgery-only in patients with resected pancreatic cancer: Japanese Study Group of Adjuvant 
Therapy for Pancreatic Cancer. Br J Cancer 101:908-915. http://doi.org/10.1038/sj.bjc.6605256.

10. Uesaka K, Boku N, Fukutomi A, et al (2016) Adjuvant chemotherapy of S-1 versus gemcitabine for resected pancreatic cancer: a phase 3 , open-label, randomised, non-inferiority trial (JASPAC 01). The Lancet 388:248-257. http://doi.org/10.1016/s0140-6736(16)30583-9.

11. Groot VP, Blair AB, Gemenetzis G, et al (2019) Recurrence after neoadjuvant therapy and resection of borderline resectable and locally advanced pancreatic cancer. Eur J Surg Oncol 45:1674-1683. http://doi.org/10.1016/j.ejso.2019.04.007.

12. Groot VP, Rezaee N, Wu W, et al (2018) Patterns, Timing, and Predictors of Recurrence Following Pancreatectomy for Pancreatic Ductal Adenocarcinoma. Ann Surg 267:936-945. http://doi.org/10.1097/SLA.0000000000002234.

13. Hattangadi JA, Hong TS, Yeap BY, Mamon HJ (2009) Results and patterns of failure in patients treated with adjuvant combined chemoradiation therapy for resected pancreatic adenocarcinoma. Cancer 115:3640-3650. http://doi.org/10.1002/cncr.24410.

14. Chang K, Pastan I (1996) Molecular cloning of mesothelin, a differentiation antigen present on mesothelium, mesotheliomas, and ovarian cancers. Proc Natl Acad Sci U S A 93:136-140. http://doi.org/10.1073/pnas.93.1.136.

15. Hassan R, Thomas A, Alewine C, Le DT, Jaffee EM, Pastan I (2016) Mesothelin Immunotherapy for Cancer: Ready for Prime Time? J Clin Oncol 34:4171-4179. http://doi.org/10.1200/JC0.2016.68.3672.

16. Tang Z, Qian M, Ho M (2013) The role of mesothelin in tumor progression and targeted therapy. Anticancer Agents Med Chem 13:276-280. http://doi.org/10.2174/1871520611313020014.

17. Yen MJ, Hsu CY, Mao TL, et al (2006) Diffuse mesothelin expression correlates with prolonged patient survival in ovarian serous carcinoma. Clin Cancer Res 12:827-831. http://doi.org/10.1158/1078-0432.CCR-05-1397.

18. Einama T, Kamachi $H$, Nishihara $H$, et al (2011) Co-expression of mesothelin and CA125 correlates with unfavorable patient outcome in pancreatic ductal adenocarcinoma. Pancreas 40:1276-1282. http://doi.org/10.1097/MPA.0b013e318221bed8.

19. Hassan R, Laszik ZG, Lerner M, Raffeld M, Postier R, Brackett D (2005) Mesothelin Is Overexpressed in Pancreaticobiliary Adenocarcinomas but Not in Normal Pancreas and Chronic Pancreatitis. American Journal of Clinical Pathology 124:838-845. http://doi.org/10.1309/f1b64cl7h8vjkeaf.

20. Gubbels JA, Belisle J, Onda M, et al (2006) Mesothelin-MUC16 binding is a high affinity, N-glycan dependent interaction that facilitates peritoneal metastasis of ovarian tumors. Mol Cancer 5:50. http://doi.org/10.1186/1476-4598-5-50.

21. Rump A, Morikawa Y, Tanaka M, et al (2004) Binding of ovarian cancer antigen CA125/MUC16 to mesothelin mediates cell adhesion. J Biol Chem 279:9190-9198. http://doi.org/10.1074/jbc.M312372200.

22. Bharadwaj U, Li M, Chen C, Yao Q (2008) Mesothelin-induced pancreatic cancer cell proliferation involves alteration of cyclin E via activation of signal transducer and activator of transcription 
protein 3. Mol Cancer Res 6:1755-1765. http://doi.org/10.1158/1541-7786.MCR-08-0095.

23. Chang MC, Chen CA, Hsieh CY, et al (2009) Mesothelin inhibits paclitaxel-induced apoptosis through the PI3K pathway. Biochem J 424:449-458. http://doi.org/10.1042/BJ20082196.

24. Cheng WF, Hung CF, Chai CY, et al (2007) Generation and characterization of an ascitogenic mesothelin-expressing tumor model. Cancer 110:420-431. http://doi.org/10.1002/cncr.22781.

25. He X, Wang L, Riedel H, et al (2017) Mesothelin promotes epithelial-to-mesenchymal transition and tumorigenicity of human lung cancer and mesothelioma cells. Mol Cancer 16:63. http://doi.org/10.1186/s12943-017-0633-8.

26. Li M, Bharadwaj U, Zhang R, et al (2008) Mesothelin is a malignant factor and therapeutic vaccine target for pancreatic cancer. Mol Cancer Ther 7:286-296. http://doi.org/10.1158/1535-7163.MCT-070483.

27. Uehara N, Matsuoka Y, Tsubura A (2008) Mesothelin promotes anchorage-independent growth and prevents anoikis via extracellular signal-regulated kinase signaling pathway in human breast cancer cells. Mol Cancer Res 6:186-193. http://doi.org/10.1158/1541-7786.MCR-07-0254.

28. Cheng WF, Huang CY, Chang MC, et al (2009) High mesothelin correlates with chemoresistance and poor survival in epithelial ovarian carcinoma. Br J Cancer 100:1144-1153. http://doi.org/10.1038/sj.bjc.6604964.

29. Einama T, Homma S, Kamachi $\mathrm{H}$, et al (2012) Luminal membrane expression of mesothelin is a prominent poor prognostic factor for gastric cancer. $\mathrm{Br} \mathrm{J}$ Cancer 107:137-142. http://doi.org/10.1038/bjc.2012.235.

30. Einama $\mathrm{T}$, Kamachi $\mathrm{H}$, Nishihara $\mathrm{H}$, et al (2015) Importance of luminal membrane mesothelin expression in intraductal papillary mucinous neoplasms. Oncol Lett 9:1583-1589. http://doi.org/10.3892/ol.2015.2969.

31. Kawamata F, Homma S, Kamachi H, et al (2014) C-ERC/mesothelin provokes lymphatic invasion of colorectal adenocarcinoma. J Gastroenterol 49:81-92. http://doi.org/10.1007/s00535-013-0773-6.

32. Kawamata F, Kamachi $H$, Einama $T$, et al (2012) Intracellular localization of mesothelin predicts patient prognosis of extrahepatic bile duct cancer. Int J Oncol 41:2109-2118. http://doi.org/10.3892/ijo.2012.1662.

33. Suzuki T, Yamagishi Y, Einama T, et al (2020) Membrane mesothelin expression positivity is associated with poor clinical outcome of luminal-type breast cancer. Oncol Lett 20:193. http://doi.org/10.3892/ol.2020.12055.

34. Hassan R, Ebel W, Routhier EL, et al (2007) Preclinical evaluation of MORAb-009, a chimeric antibody targeting tumor-associated mesothelin. Cancer Immun 7:20.

35. Feng Y, Xiao X, Zhu Z, et al (2009) A novel human monoclonal antibody that binds with high affinity to mesothelin-expressing cells and kills them by antibody-dependent cell-mediated cytotoxicity. Mol Cancer Ther 8:1113-1118. http://doi.org/10.1158/1535-7163.MCT-08-0945.

36. Fujimori K, Covell DG, Fletcher JE, Weinstein JN (1990) A modeling analysis of monoclonal antibody percolation through tumors: a binding-site barrier. J Nucl Med 31:1191-1198. 
37. Lindenberg L, Thomas A, Adler S, et al (2015) Safety and biodistribution of 111 In-amatuximab in patients with mesothelin expressing cancers using single photon emission computed tomographycomputed tomography (SPECT-CT) imaging. Oncotarget 6:4496-4504. http://doi.org/10.18632/oncotarget.2883.

38. Uzunparmak B, Sahin IH (2019) Pancreatic cancer microenvironment: a current dilemma. Clin Transl Med 8:2. http://doi.org/10.1186/s40169-019-0221-1.

39. Shin IS, Lee SM, Kim HS, et al (2011) Effect of chelator conjugation level and injection dose on tumor and organ uptake of $111 \mathrm{In}$-labeled MORAb-009, an anti-mesothelin antibody. Nucl Med Biol 38:11191127. http://doi.org/10.1016/j.nucmedbio.2011.05.003.

40. Lee JH, Kim H, Yao Z, et al (2015) Tumor and organ uptake of (64)Cu-labeled MORAb-009 (amatuximab), an anti-mesothelin antibody, by PET imaging and biodistribution studies. Nucl Med Biol 42:880-886. http://doi.org/10.1016/j.nucmedbio.2015.07.008.

41. Mizukami T, Kamachi H, Fujii Y, et al (2018) The anti-mesothelin monoclonal antibody amatuximab enhances the anti-tumor effect of gemcitabine against mesothelin-high expressing pancreatic cancer cells in a peritoneal metastasis mouse model. Oncotarget 9:33844-33852. http://doi.org/10.18632/oncotarget.26117.

42. Matsuzawa F, Kamachi $\mathrm{H}$, Mizukami T, et al (2021) Mesothelin blockage by Amatuximab suppresses cell invasiveness, enhances gemcitabine sensitivity and regulates cancer cell stemness in mesothelin-positive pancreatic cancer cells. BMC Cancer 21:200. http://doi.org/10.1186/s12885020-07722-3.

43. Flavell DJ, Warnes SL, Bryson CJ, et al (2006) The anti-CD20 antibody rituximab augments the immunospecific therapeutic effectiveness of an anti-CD19 immunotoxin directed against human Bcell lymphoma. Br J Haematol 134:157-170. http://doi.org/10.1111/j.1365-2141.2006.06155.x.

44. Nishihara T, Sawada T, Yamamoto A, et al (2000) Antibody-dependent cytotoxicity mediated by chimeric monoclonal antibody $\mathrm{Nd} 2$ and experimental immunotherapy for pancreatic cancer. Jpn $\mathrm{J}$ Cancer Res 91:817-824. http://doi.org/10.1111/j.1349-7006.2000.tb01019.x.

45. Dekkers G, Bentlage AEH, Stegmann TC, et al (2017) Affinity of human IgG subclasses to mouse Fc gamma receptors. MAbs 9:767-773. http://doi.org/10.1080/19420862.2017.1323159.

46. Ng SS, Tsao MS, Nicklee T, Hedley DW (2001) Wortmannin inhibits pkb/akt phosphorylation and promotes gemcitabine antitumor activity in orthotopic human pancreatic cancer xenografts in immunodeficient mice. Clin Cancer Res 7:3269-3275.

47. Ng SSW, Tsao MS, Chow S, Hedley DW (2000) Inhibition of phosphatidylinositide 3-kinase enhances gemcitabine-induced apoptosis in human pancreatic cancer cells. Cancer Res 60:5451-5455.

48. Fujisaka Y, Kurata T, Tanaka K, et al (2015) Phase I study of amatuximab, a novel monoclonal antibody to mesothelin, in Japanese patients with advanced solid tumors. Invest New Drugs 33:380388. http://doi.org/10.1007/s10637-014-0196-0.

\section{Supplemental Figures}




\section{Figures}
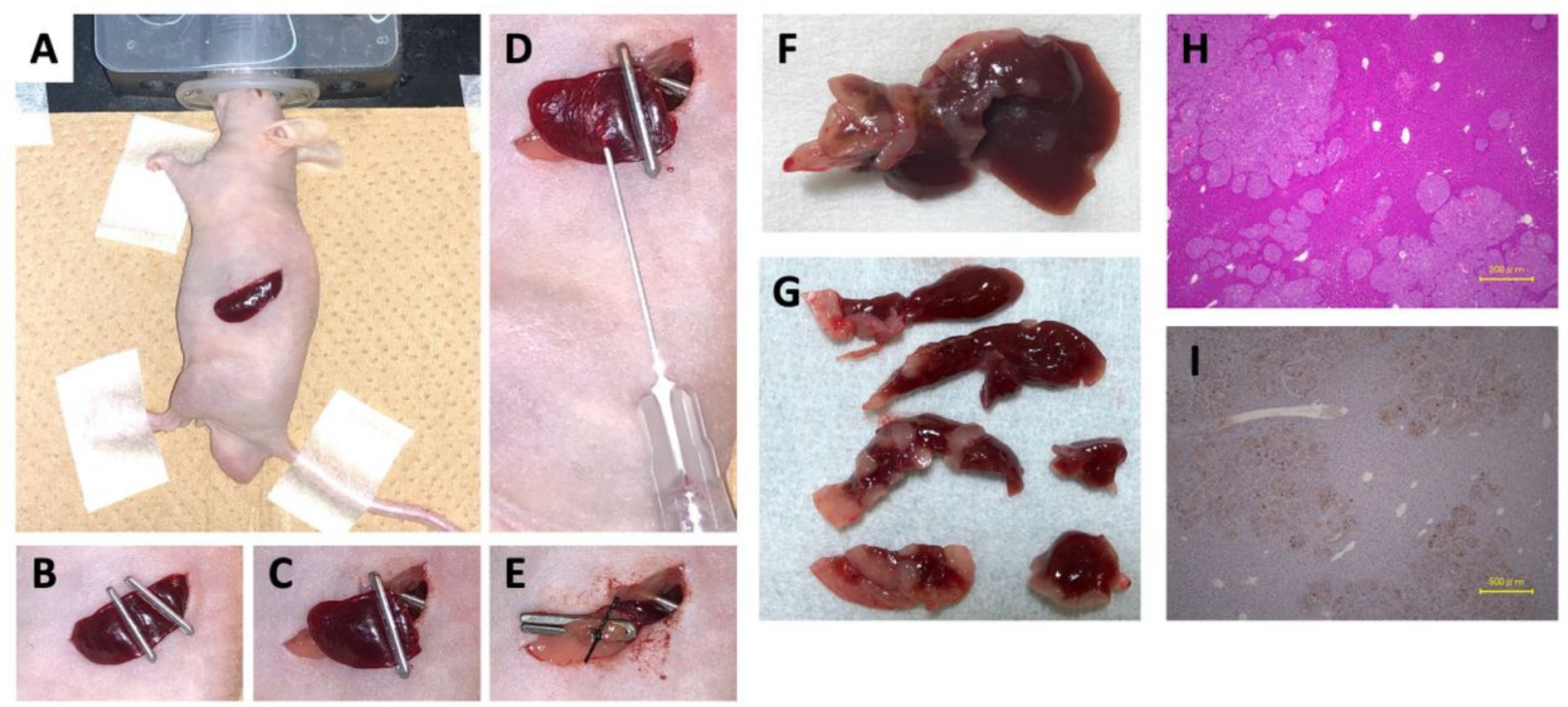

\section{Figure 1}

Hemi-splenic injection method. (A) Laparotomy was performed by left subcostal incision along the spleen under general anesthesia induced by $3 \%$ isoflurane. We extracted the spleen and dissected the avascular area of the splenic hilum. (B) The spleen was dissected on the midline after clipping. (C) Then, we restored the cranial half into the abdominal cavity. (D) We injected $100 \mu l$ cell suspension into the splenic parenchyma using a $30 \mathrm{G}$ needle and held up the spleen for $2 \mathrm{~min}$ to allow the cells to pass through. (E) After that, we clipped the hilar blood vessels and removed the caudal spleen. We irrigated the intraabdominal cavity with $20 \mathrm{~mL}$ sterile normal saline and made a running suture of the fascia and skin. The wound was covered by surgical tape. (F) Gross finding of liver metastases. (G) HE staining of liver revealed multi-centric metastatic lesions. $(\mathrm{H})$ The metastatic lesions were positive for mesothelin by immunohistochemical staining with anti-mesothelin antibody. 
(A)

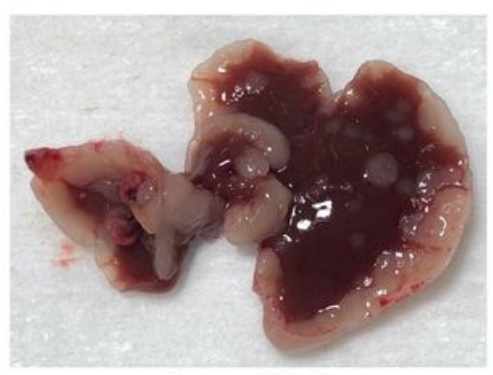

Control IgG

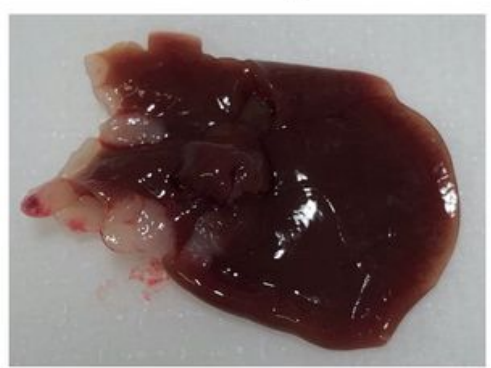

Amatuximab
(B)

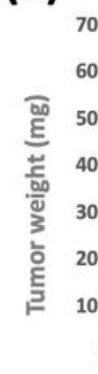

(C)
(D)

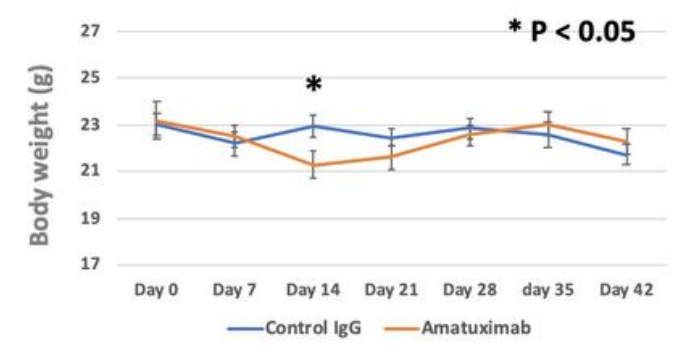

\section{Figure 2}

(A) Gross findings of liver metastases in the control IgG group and amatuximab group. The control IgG group formed obviously more liver metastases than the amatuximab group $(n=8)$. (B) The total weight of liver metastases of mice in the amatuximab group was significantly lower than that in the control group (100.1 $\pm 52.4 \mathrm{mg}$ vs. $489.1 \pm 164.9 \mathrm{mg}, \mathrm{P}=0.041)$. (C) Serum Gluc activity on days 21,35 , and 42 was significantly higher in the control group than in the amatuximab group; $14321.3 \pm 1134.4 \mathrm{RLU}$ vs. $10899 \pm 710.2 \mathrm{RLU}(P=0.028), 41705 \pm 10875.3 \mathrm{RLU}$ vs. $16282.6 \pm 2946.9 \mathrm{RLU}(\mathrm{P}=0.041)$, and $90350 \pm$ 31267.6 RLU vs. $22282.9 \pm 5170.1$ RLU ( $P=0.0497)$, respectively. (D) Body weights of mice in the two groups were equivalent $(P>0.05$ at all points except on day $14 ; 23.0 \pm 0.38 \mathrm{~g}$ in the control IgG group vs. $21.3 \pm 0.57 \mathrm{~g}$ in the amatuximab group, $\mathrm{P}=0.039$ ). 


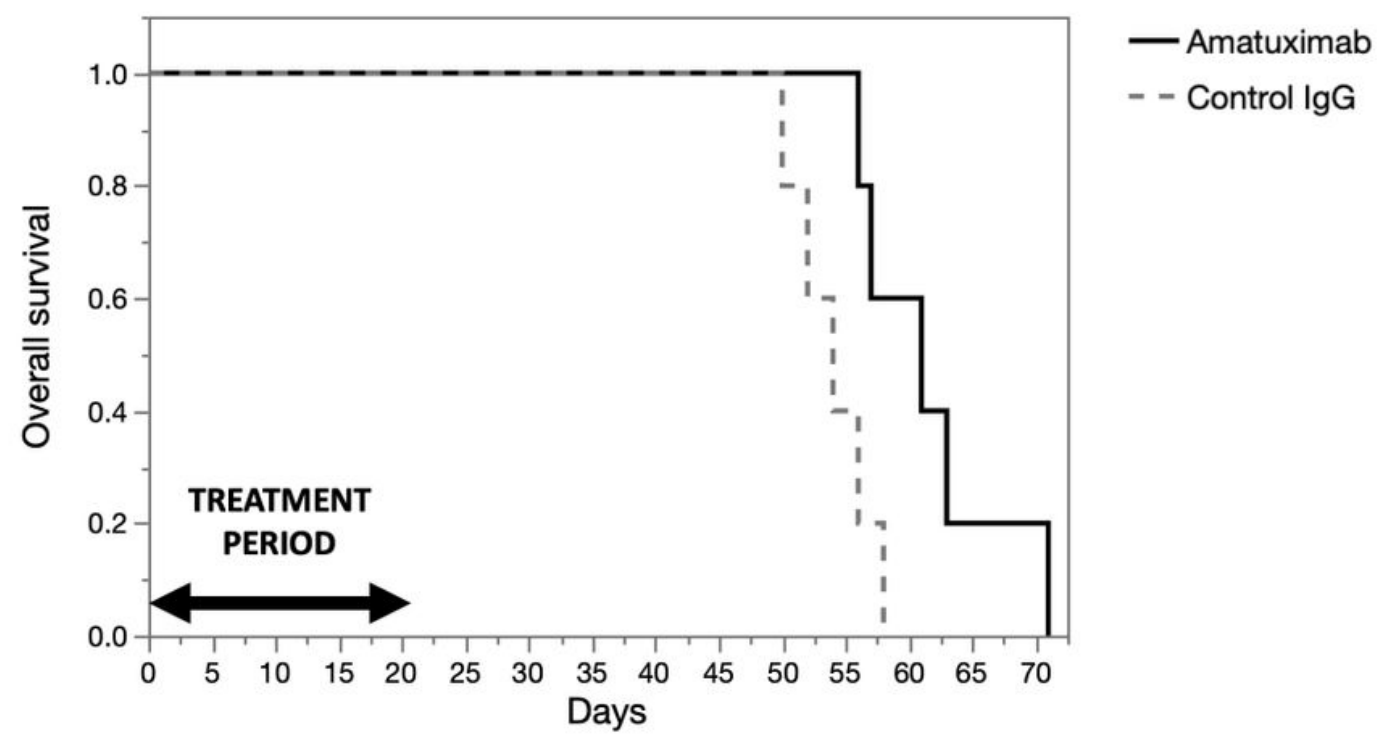

\section{Figure 3}

Tridaily amatuximab or control IgG treatment was initiated in the liver metastasis mouse models on the same day of tumor inoculation $(n=5)$. The treatment period was set for 3 weeks. Mice in the amatuximab group survived longer than those in the control group. The median OS of mice in the two groups was 61 days in the control IgG group and 54 days in the amatuximab group $(P=0.0256)$. 
(A)

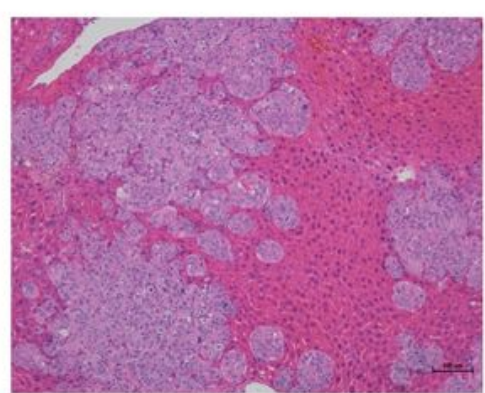

Control IgG

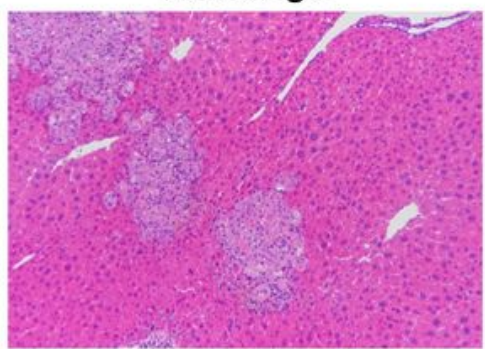

Control IgG + GEM

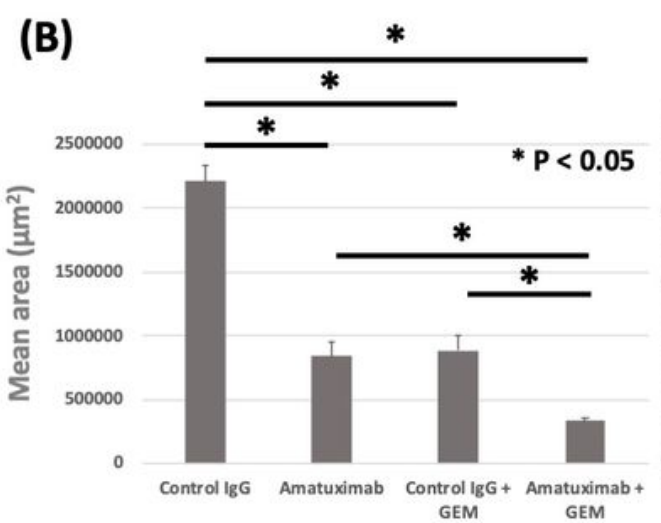

(D)

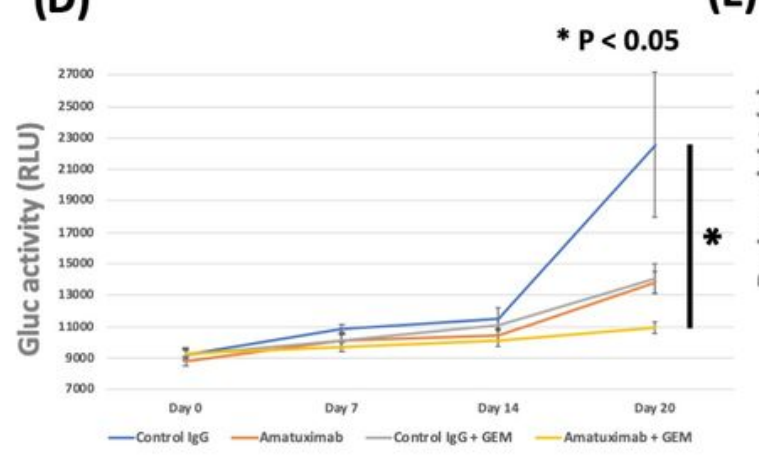

(E)
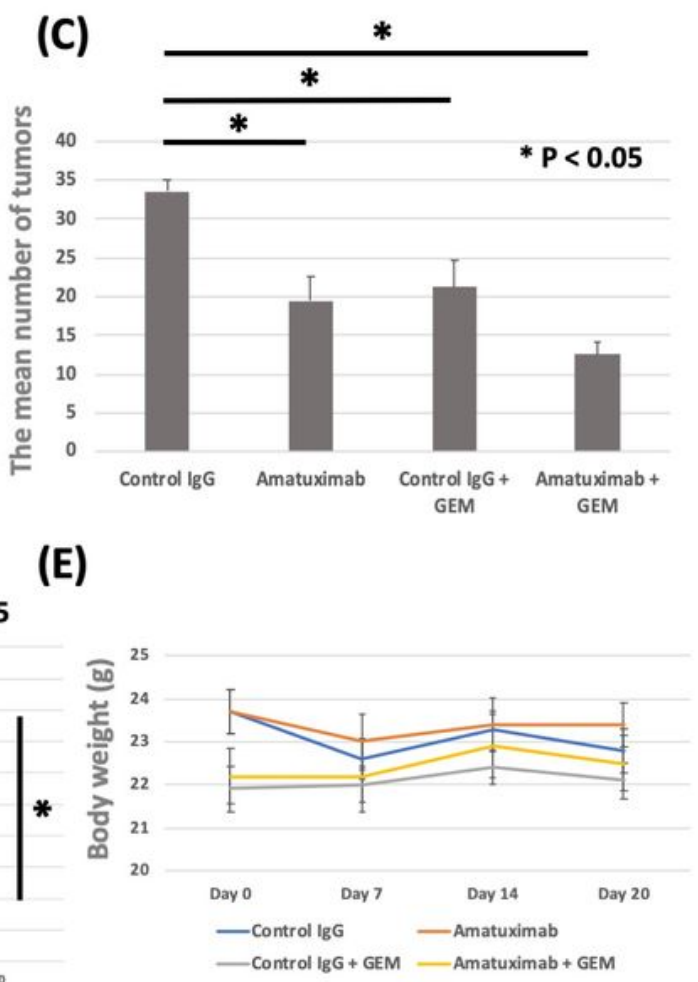

Figure 4

(A) Representative H\&E findings demonstrated the greatest liver metastases in the control group and the smallest in the amatuximab + GEM group. (B) On ImageJ analysis, the metastatic tumors in the three treatment groups of amatuximab, control IgG + GEM, and amatuximab + GEM were significantly smaller in area than those in the control IgG group $(8.4 \pm 1.2 \times 105 \mu \mathrm{m} 2,8.9 \pm 1.1 \times 105 \mu \mathrm{m} 2,3.4 \pm 0.3 \times 105 \mu \mathrm{m} 2$, and $22.1 \pm 1.2 \times 105 \mu \mathrm{m} 2$, respectively; $\mathrm{P}<0.0001)$. Furthermore, of these three treatment groups, the 
amatuximab + GEM group exhibited the significantly smallest tumor area. (C) The number of tumors in the three treatment groups was significantly lower than that in the control IgG group $(19.5 \pm 3.0,21.2 \pm$ $3.5,12.5 \pm 1.7$, and $33.5 \pm 1.5$, respectively; $P<0.0001$ ). (D) Serum Gluc activity in the amatuximab + GEM group was significantly lower than that in the control group on day 20 (10910 \pm 383.6 RLU vs. $22566.7 \pm 4610.8 \mathrm{RLU}, P=0.012)$. (E) The average body weights of mice in all groups were equivalent $(P$ $\geq 0.05$ at all points).

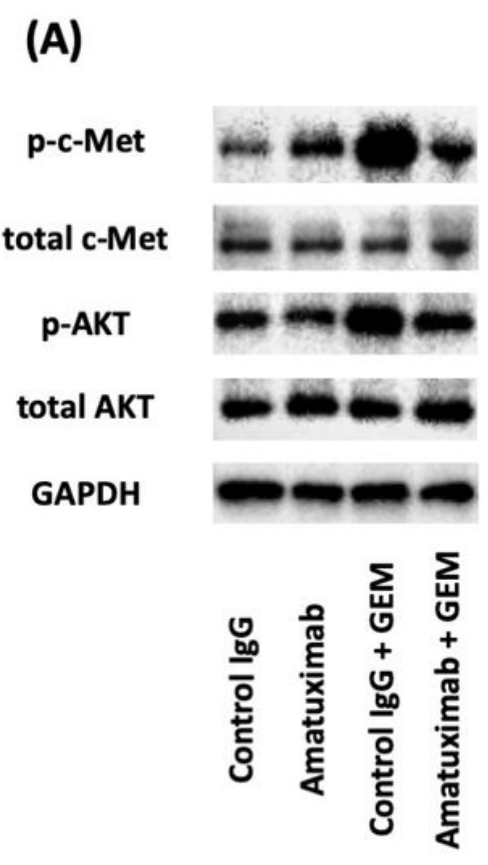

(B)

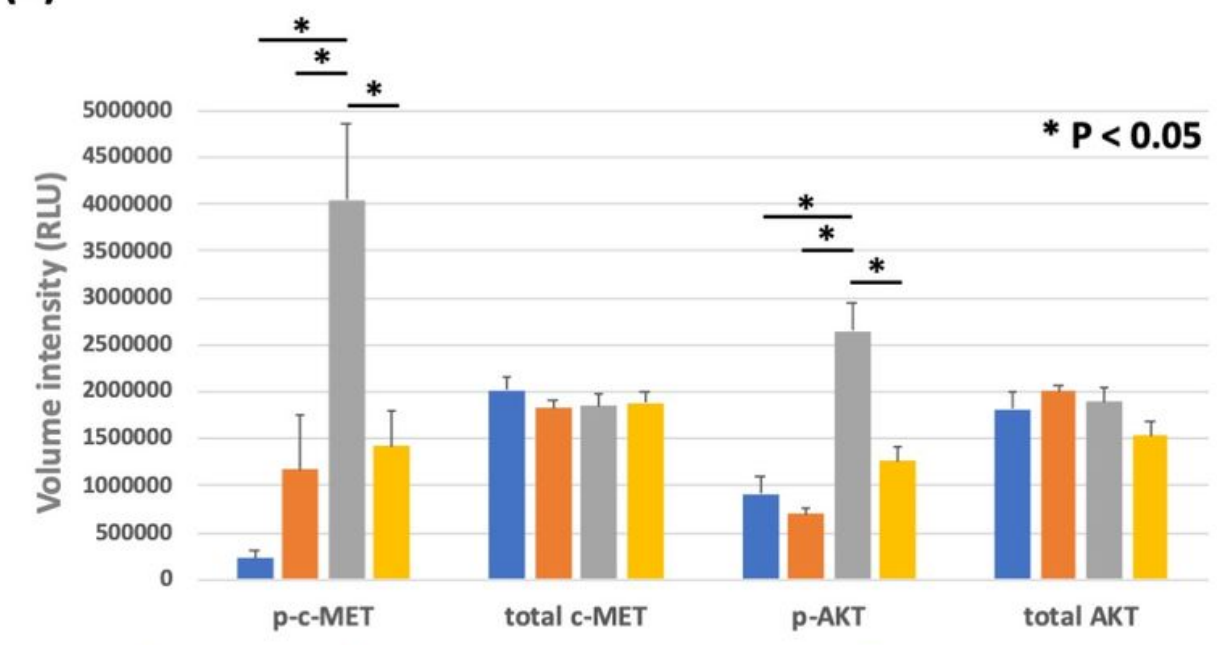

n Control IgG $=$ Amatuximab $=$ Control IgG + GEM $\approx$ Amatuximab + GEM

\section{Figure 5}

(A) Protein expression of c-Met and AKT in the liver metastases of each treatment group was evaluated by western blotting. The results showed significantly higher levels of phosphorylated c-Met and AKT in the control IgG + GEM group than in the other groups. However, the expression of total c-Met and AKT was equivalent among all four groups. (B) These results were confirmed by quantification of blotting data. Protein lysates were extracted from the liver metastases of all mice in each group $(n=6)$. The quantified values of phosphorylated c-Met and AKT were significantly higher in the control IgG + GEM group than in the other groups. The mean values of phosphorylated c-Met and AKT in each group were as follows: $226573.3 \pm 74141.5 \mathrm{RLU}$ and $916478 \pm 190552.5 \mathrm{RLU}$ in the control IgG group, $1171036.6 \pm$ 585276.0 RLU and 692315.3 $\pm 65489.7 \mathrm{RLU}$ in the amatuximab group, 4049143.7 $\pm 811280.6 \mathrm{RLU}$ and $2643160 \pm 300584.1$ RLU in the control IgG + GEM group, and 1415822.7 \pm 390206.7 RLU and 1264898.6 $\pm 141429.7 \mathrm{RLU}$ in the amatuximab + GEM group. 